Supporting Information

\title{
Ultrafast Carrier Dynamics in 2D CdSe Nanoplatelets-CsPbX 3 Composites: Influence of Halide Composition
}

\author{
Goutam Ghosh, Avisek Dutta, Arnab Ghosh, Srijon Ghosh, and Amitava Patra ${ }^{1 *}$ \\ School of Materials Sciences, Indian Association for the Cultivation of Science, Jadavpur, \\ Kolkata-700032, India \\ ${ }^{1}$ Institute of Nano Science and Technology, Habitat Centre, Sector 64, Phase 10, Mohali 160062, \\ India
}

*To whom correspondence should be addressed. E-mail: $\underline{\text { msap@,iacs.res.in }}$

Phone: (91)-33-2473-4971, Fax: (91)-33-2473-2805 


\section{Calculation of PL quantum yield (PLQY):}

The PLQY of all $\mathrm{CsPbX}_{3} \mathrm{NCs}$ and CdSe NPLs are measured with respect to reference dye quinine sulphate in $(0.1 \mathrm{M}) \mathrm{H}_{2} \mathrm{SO}_{4}$ by using the following equation:

$\phi_{\mathrm{s}}=\phi_{\mathrm{r}} \frac{\mathrm{F}_{\mathrm{s}} \mathrm{OD} \mathrm{D}_{\mathrm{r}}^{2}}{\mathrm{~F}_{\mathrm{r}} \mathrm{OD} \eta_{\mathrm{s}}^{2}}$

Where $\phi_{\mathrm{s}}$ represents measured PLQY of the actual sample, $\phi_{\mathrm{r}}$ represents standard PLQY of quinine sulphate $(0.53)$ in $0.1 \mathrm{M} \mathrm{H}_{2} \mathrm{SO}_{4}, \mathrm{OD}_{\mathrm{r}}$ and $\mathrm{OD}_{\mathrm{s}}$ represents actual absorbance of reference and sample respectively at $370 \mathrm{~nm} . \mathrm{F}_{\mathrm{S}} \mathrm{AndF}_{\mathrm{r}}$ represent integrated area of sample and reference, excited at $370 \mathrm{~nm}$ respectively. $\eta$ refers to the refractive index of solvent medium.

\section{Calculation of concentrations of $\mathrm{CsPbX}_{3} \mathrm{NCs}$ :}

Concentration of $\mathrm{CsPbX}_{3} \mathrm{NCs}$ are measured following method described by Nag et al. ${ }^{1}$ Briefly, each $\mathrm{CsPbX}_{3}$ NCs are divided into two parts and centrifuged at 700 r.p.m; one part is dissolved into fixed volume of toluene and another part is vacuum dried to get the weight of the nanocrystal. We first measured the weight of single nanocrystal (NC) by using $\mathrm{m}=\mathrm{d} / \mathrm{V}$, where $\mathrm{m}$ is the mass of single $\mathrm{NC}, \mathrm{V}$ is the volume of a $\mathrm{NC}\left(\mathrm{V}=\mathrm{a}^{3}\right.$, $\mathrm{a}$ is the average edge length measure from TEM), and $\mathrm{d}$ is the density of bulk $\mathrm{CsPbX}_{3}\left(\mathrm{CsPbBr}_{3}=4.86 \mathrm{~g} / \mathrm{cm}^{3}, \mathrm{CsPbI}_{3}=5.07 \mathrm{~g} / \mathrm{cm}^{3}\right) .^{2} \mathrm{It}$ is worth noting that densities for $\mathrm{CsPbBr}_{1.5} \mathrm{I}_{1.5}$ and $\mathrm{CsPbrI}_{2}$ are calculated from $\mathrm{CsPbBr}_{3}$ and $\mathrm{CsPbI}_{3}$ after weighing with the composition of the alloy. Then, total number of $\mathrm{NC}$ in particular sample is obtained by dividing the total weight of the inorganic part of the sample (i.e. subtracting the contribution of organic ligands) with mass of a single NC. Now, moles of the NC are obtained by dividing the number with Avogadro's number. Finally, knowing the volume of the solvent we calculated molar concentration of the NC. Now, molar extinction coefficient $(\varepsilon)$ for each $\mathrm{CsPbX}_{3} \mathrm{NCs}$ are found from Beer-Lambert Law by using $\mathrm{A}=\varepsilon \times \mathrm{C} \times \mathrm{L}$; where $\mathrm{A}$ is absorbance, $\mathrm{C}$ is molar concentration and $\varepsilon$ is the molar extinction coefficient. 

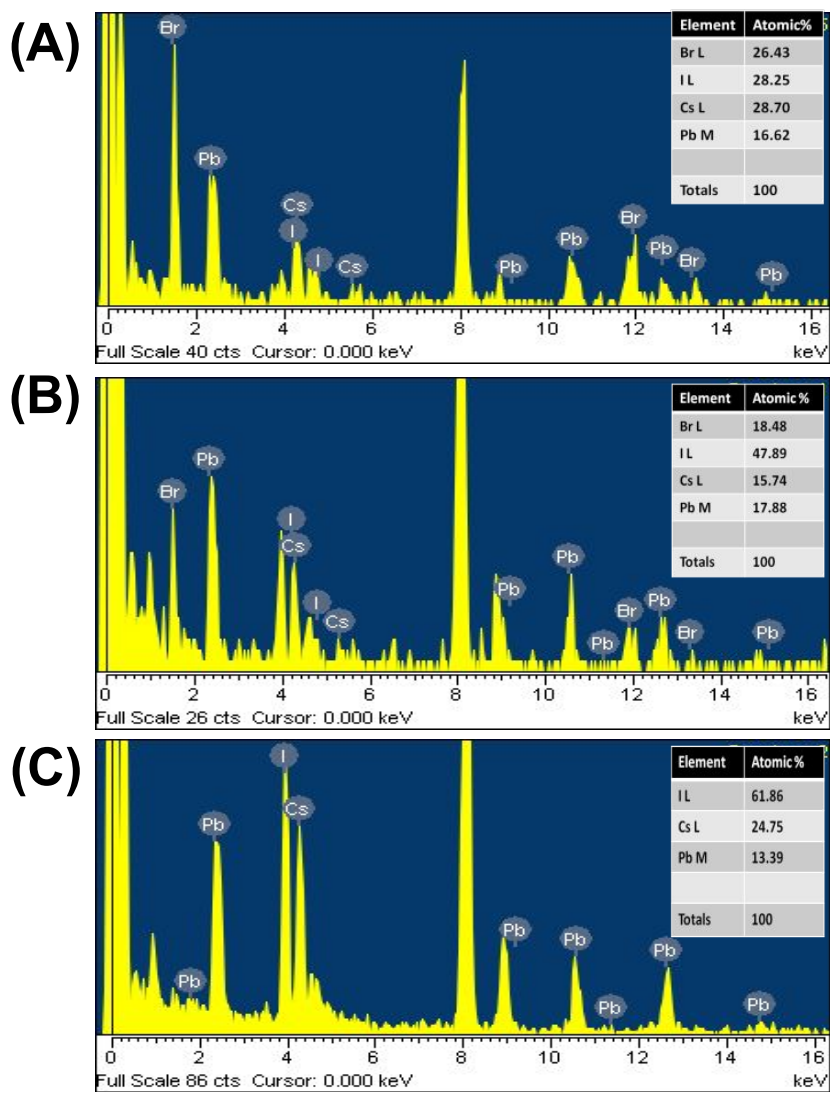

Figure S1. EDS spectra and corresponding elemental analysis table (inset) of (A) $\mathrm{CsPbBr}_{1.5} \mathrm{I}_{1.5}$, (B) $\mathrm{CsPbBrI}_{2}$ and (C) $\mathrm{CsPbI}_{3} \mathrm{NCs}$.

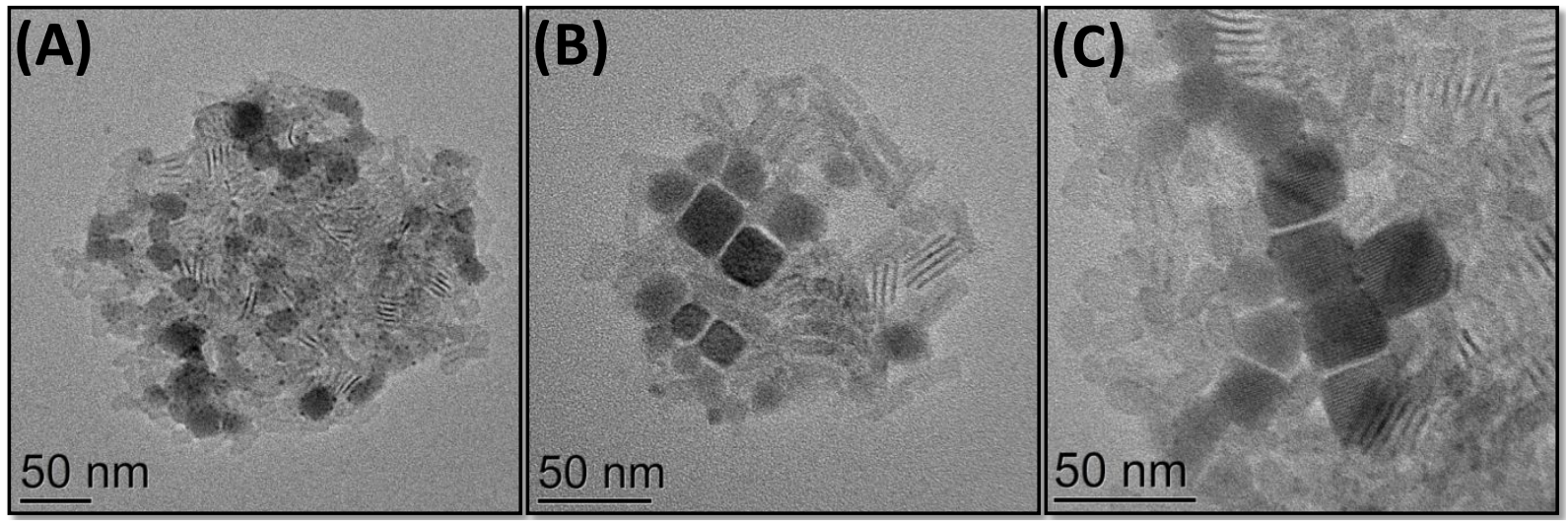

Figure S2. TEM images of composites (A) CdSe-CsPbBr ${ }_{1.5} \mathrm{I}_{1.5}$, (B) CdSe-CsPbBrI 2 and (C) CdSe$\mathrm{CsPbI}_{3}$. 
Table S1. Time resolved PL decay parameters of the pure CdSe NPLs and corresponding composites.

\begin{tabular}{|c|c|c|c|c|}
\hline Systems & $\tau_{1}^{x}\left(a_{1}\right)^{y}$ & $\tau_{2}{ }^{x}\left(a_{2}\right)^{y}$ & $\tau_{3}{ }^{x}\left(a_{3}\right)^{y}$ & $\tau_{\text {average }}$ \\
\hline & (ns) & (ns) & (ns) & (ns) \\
\hline \multirow[t]{2}{*}{ CdSe NPLs } & 0.5 & 2.9 & 16.2 & $1.2 \pm 0.23$ \\
\hline & $(0.85 \%)$ & $(0.12 \%)$ & $(0.03 \%)$ & \\
\hline \multirow[t]{2}{*}{$\mathrm{CdSe}-\mathrm{CsPbBr}{ }_{1.5} \mathrm{I}_{1.5}$} & 0.35 & 2.9 & 9.8 & $0.71 \pm 0.13$ \\
\hline & $(0.91 \%)$ & $(0.07 \%)$ & $(0.02 \%)$ & \\
\hline \multirow[t]{2}{*}{$\mathrm{CdSe}-\mathrm{Cs} \mathrm{PbBrI}_{2}$} & 0.30 & 1.9 & 8.7 & $0.55 \pm 0.11$ \\
\hline & $(0.93 \%)$ & $(0.05 \%)$ & $(0.02 \%)$ & \\
\hline \multirow[t]{2}{*}{$\mathrm{CdSe}-\mathrm{CsPbI}{ }_{3}$} & 0.26 & 1.3 & 7.1 & $0.37 \pm 0.08$ \\
\hline & $(0.95 \%)$ & $(0.04 \%)$ & $(0.01 \%)$ & \\
\hline $\mathrm{x}_{ \pm} 4 \%$ and $\mathrm{y}_{ \pm} 5 \%$ & & & & \\
\hline
\end{tabular}


(A)

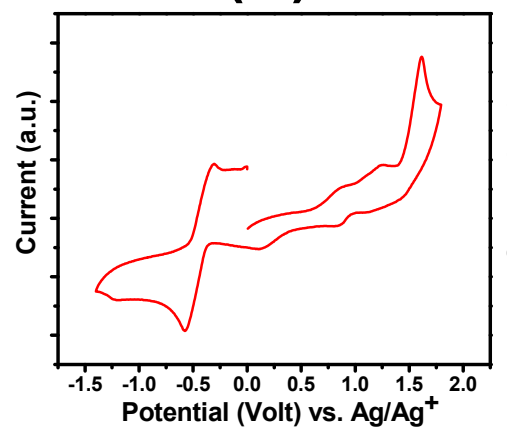

(B)

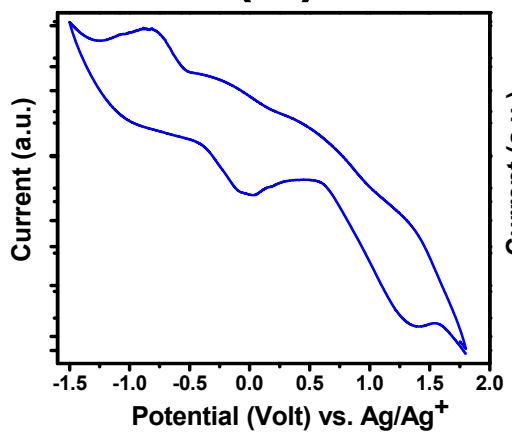

(C)

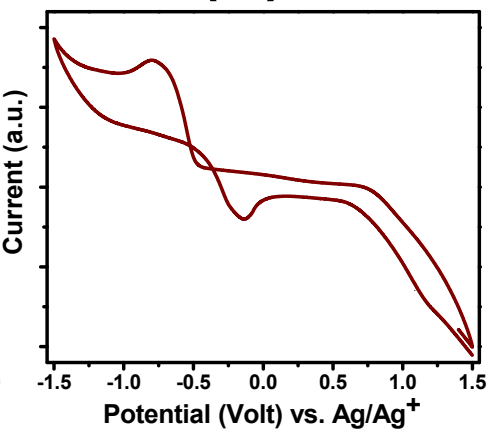

Figure S3. Cyclic voltammetric (CV) curves of (A) $\mathrm{CsPbBr}_{1.5} \mathrm{I}_{1.5}$, (B) $\mathrm{CsPbBr}_{2}$ and (C) $\mathrm{CsPbI}_{3}$ $\mathrm{NCs}$ with respect to $\mathrm{Ag} / \mathrm{Ag}+$ electrodes.

(A)

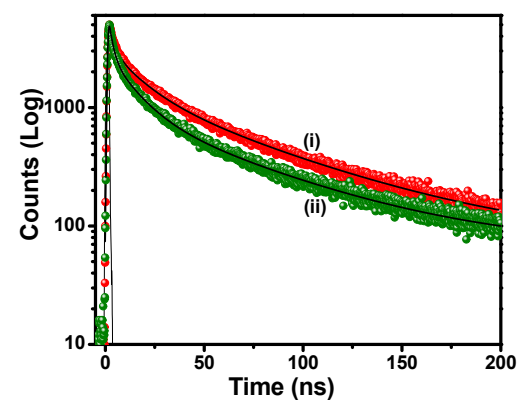

(B)

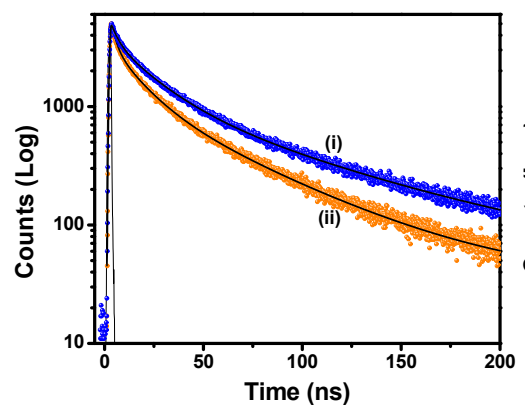

(C)

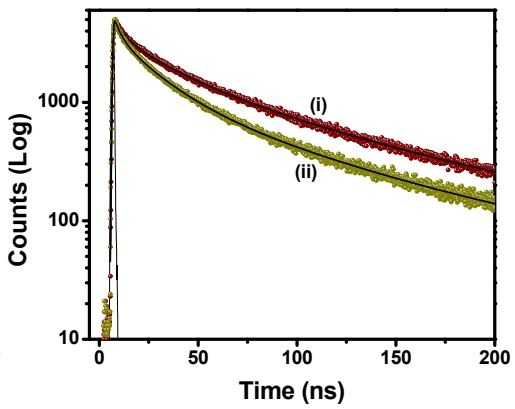

Figure S4. PL decay profiles of (A) $\mathrm{CsPbBr}_{1.5} \mathrm{I}_{1.5} \mathrm{NCs}$ (i), CdSe-CsPbBr $\mathrm{Pb}_{1.5} \mathrm{I}_{1.5}$ (ii), (B) $\mathrm{CsPbBr}_{2}$

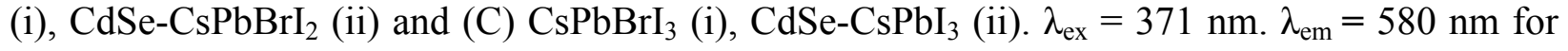
(A), $635 \mathrm{~nm}$ for (B) and $688 \mathrm{~nm}$ for (C). 
Table S2. Fluorescence up-conversion decay parameters of pure CdSe NPLs and corresponding composites with $\mathrm{CsPbX}_{3} \mathrm{NCs}$.

\begin{tabular}{|c|c|c|c|}
\hline Systems & $\begin{array}{c}\tau_{1}\left(\mathbf{a}_{1} \%\right) \\
(\mathbf{p s})\end{array}$ & $\begin{array}{c}\tau_{2}\left(\mathbf{a}_{2} \%\right) \\
(\mathbf{p s})\end{array}$ & $\begin{array}{c}\tau_{3}\left(\mathbf{a}_{3} \%\right) \\
(\mathbf{p s})\end{array}$ \\
\hline Cdse NPLs & $\begin{array}{c}29.7 \pm 0.5 \mathrm{ps} \\
(56 \%)\end{array}$ & $\begin{array}{c}>100 \text { ps } \\
(44 \%)\end{array}$ & - \\
\hline $\mathrm{CdSe}-\mathrm{CsPbBr}{ }_{1.5} \mathrm{I}_{1.5}$ & $\begin{array}{c}6.4 \pm 0.15 \mathrm{ps} \\
(36 \%)\end{array}$ & $\begin{array}{c}17.9 \pm 0.35 \mathrm{ps} \\
(46 \%)\end{array}$ & $\begin{array}{r}>100 \text { ps } \\
(18 \%)\end{array}$ \\
\hline $\mathrm{CdSe}-\mathrm{CsPbBrI}{ }_{2}$ & $\begin{array}{c}2.7 \pm 0.1 \mathrm{ps} \\
(40 \%)\end{array}$ & $\begin{array}{c}15.2 \pm 0.26 \mathrm{ps} \\
(45 \%)\end{array}$ & $\begin{array}{c}>100 \mathrm{ps} \\
(15 \%)\end{array}$ \\
\hline $\mathrm{CdSe}-\mathrm{CsPbI}{ }_{3}$ & $\begin{array}{c}1.6 \pm 0.08 \mathrm{ps} \\
(48 \%)\end{array}$ & $\begin{array}{c}12.3 \pm 0.20 \mathrm{ps} \\
(40 \%)\end{array}$ & $\begin{array}{c}>100 \mathrm{ps} \\
(12 \%)\end{array}$ \\
\hline
\end{tabular}


(A)

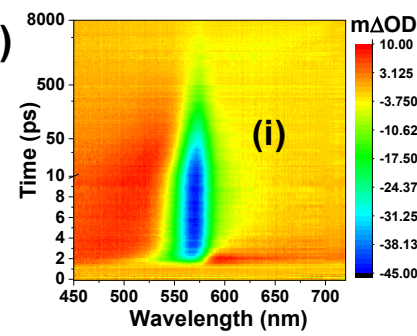

(B)

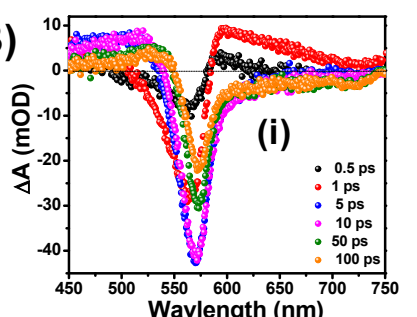

(C)

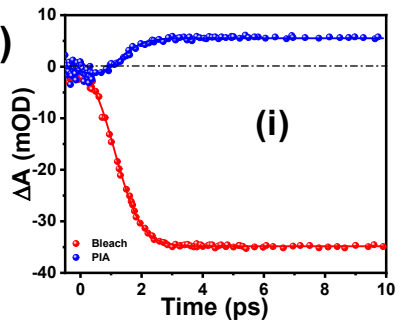

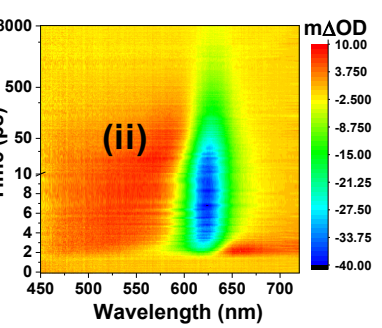
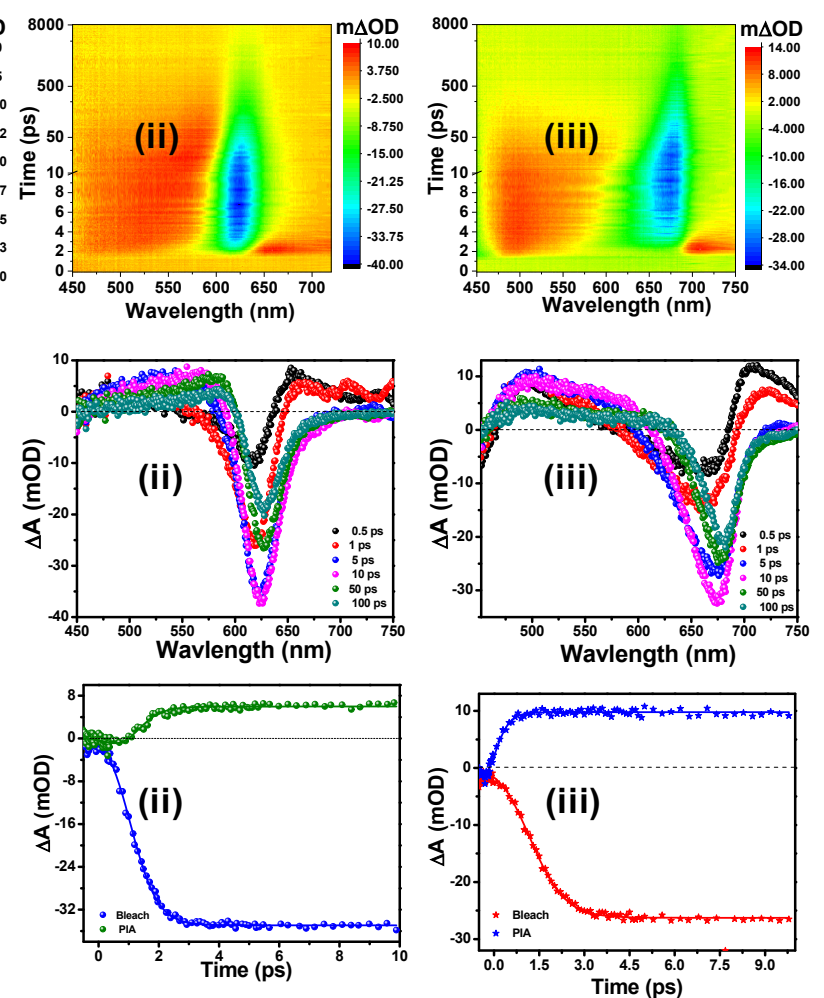

Figure S5.(A) 2D heat map, (B) TA spectral profiles, and (C) TA dynamics (EB and PIA) of pure (i) $\mathrm{CsPbBr}_{1.5} \mathrm{I}_{1.5}$, (ii) $\mathrm{CsPbBrI}_{2}$ and (iii) $\mathrm{CsPbI}_{3} \mathrm{NCs}$. 
Table S3. Fitted kinetics parameters of pure CdSe NPLs and CdSe-CsPbX ${ }_{3}$ composites at $513 \mathrm{~nm}$.

\begin{tabular}{|c|c|c|c|c|}
\hline System & $\tau_{g}\left(\mathbf{a}_{g} \%\right)$ & $\tau_{1}\left(\mathbf{a}_{1} \%\right)$ & $\tau_{2}\left(\mathbf{a}_{2} \%\right)$ & $\tau_{3}\left(\mathbf{a}_{3} \%\right)$ \\
\hline CdSe NPLs & $\begin{array}{l}800 \pm 0.5 \mathrm{fs} \\
(100 \%)\end{array}$ & $\begin{array}{l}9.3 \pm 0.2 \mathrm{ps} \\
(15 \%)\end{array}$ & $\begin{array}{l}290 \pm 0.85 \mathrm{ps} \\
(40 \%)\end{array}$ & $\begin{array}{l}>1 \mathrm{~ns} \\
(45 \%)\end{array}$ \\
\hline Cdse-CsPbBr ${ }_{1.5} \mathrm{I}_{1.5}$ & $\begin{array}{l}<100 \text { fs } \\
(100 \%)\end{array}$ & $\begin{array}{l}3.8 \pm 0.15 \text { ps } \\
(30 \%)\end{array}$ & $\begin{array}{l}197 \pm 0.72 \mathrm{ps} \\
(31 \%)\end{array}$ & $\begin{array}{l}>1 \mathrm{~ns} \\
(39 \%)\end{array}$ \\
\hline $\mathrm{CdSe}-\mathrm{CsPbBrI} 2$ & $\begin{array}{l}<100 \mathrm{fs} \\
(100 \%)\end{array}$ & $\begin{array}{l}2.1 \pm 0.10 \mathrm{ps} \\
(41 \%)\end{array}$ & $\begin{array}{l}179 \pm 0.55 \mathrm{ps} \\
(23 \%)\end{array}$ & $\begin{array}{l}>1 \mathrm{~ns} \\
(36 \%)\end{array}$ \\
\hline $\mathrm{CdSe}-\mathrm{CsPbI}{ }_{3}$ & $\begin{array}{l}<100 \mathrm{fs} \\
(100 \%)\end{array}$ & $\begin{array}{l}1.4 \pm 0.10 \mathrm{ps} \\
(48 \%)\end{array}$ & $\begin{array}{l}150 \pm 0.46 \text { ps } \\
(19 \%)\end{array}$ & $\begin{array}{l}>1 \mathrm{~ns} \\
(33 \%)\end{array}$ \\
\hline
\end{tabular}

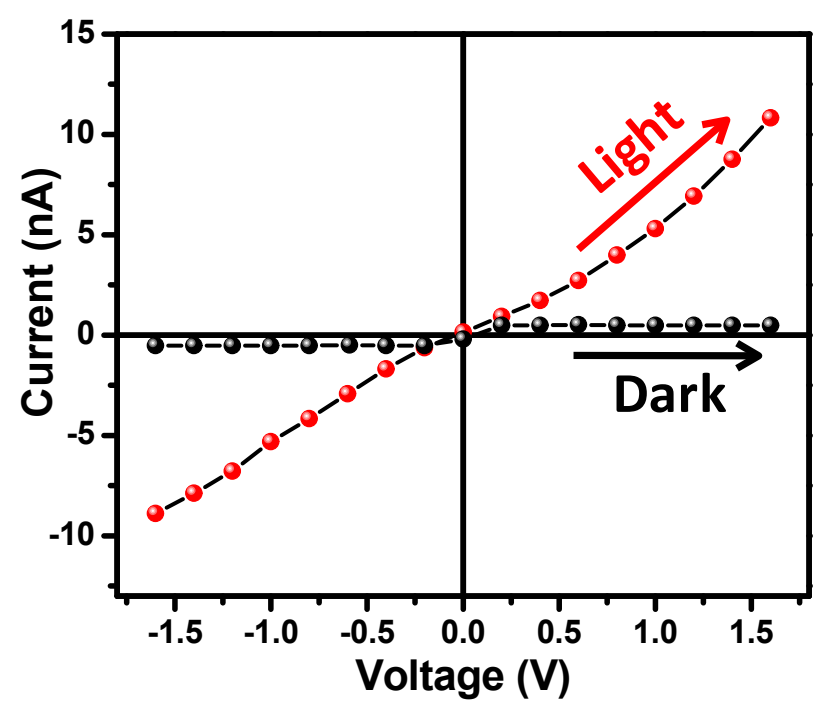

Figure S6. I-V curves of pure CdSe NPLs. 
(A)

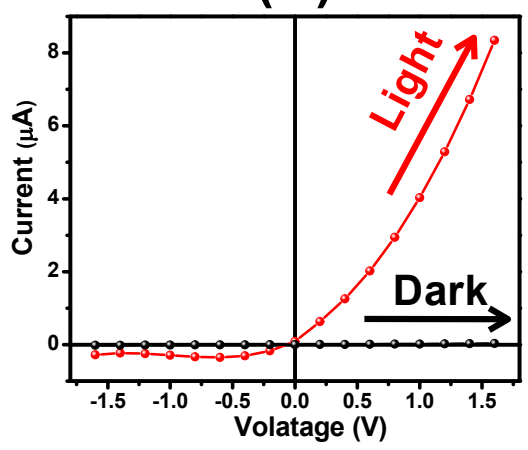

(B)

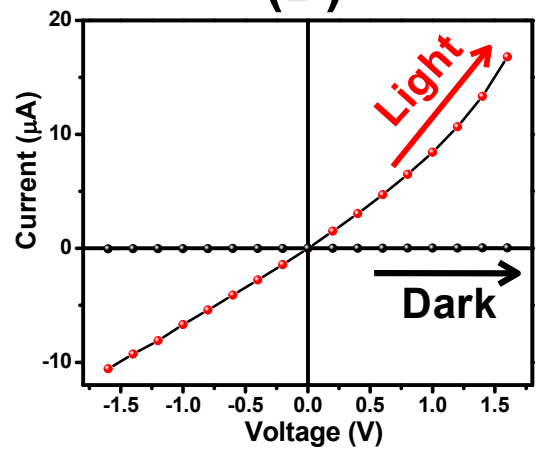

(C)

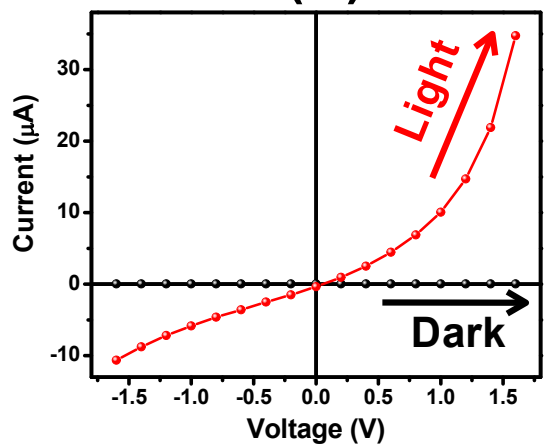

Figure S7. I-V curves of pure (A) $\mathrm{CsPbBr}_{1.5} \mathrm{I}_{1.5} \mathrm{NCs}$, (B) $\mathrm{CsPbBrI}_{2} \mathrm{NCs}$ and (C) $\mathrm{CsPbI}_{3} \mathrm{NCs}_{\text {. }}$

(A)

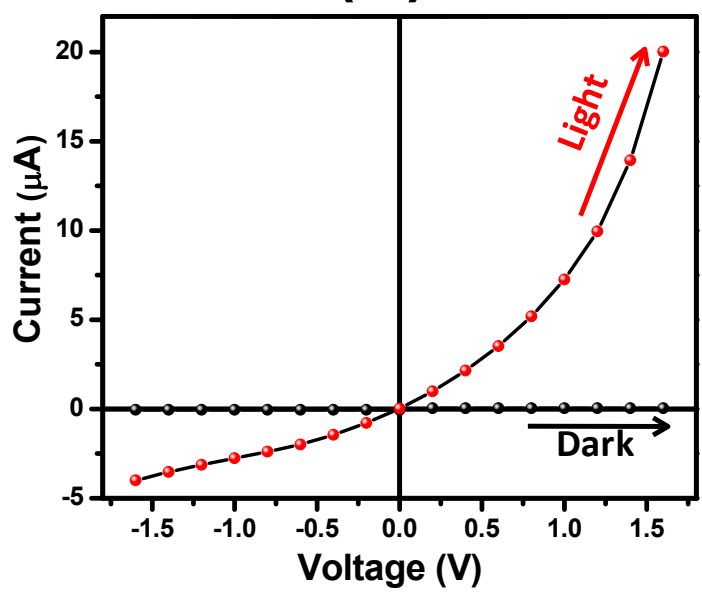

(B)

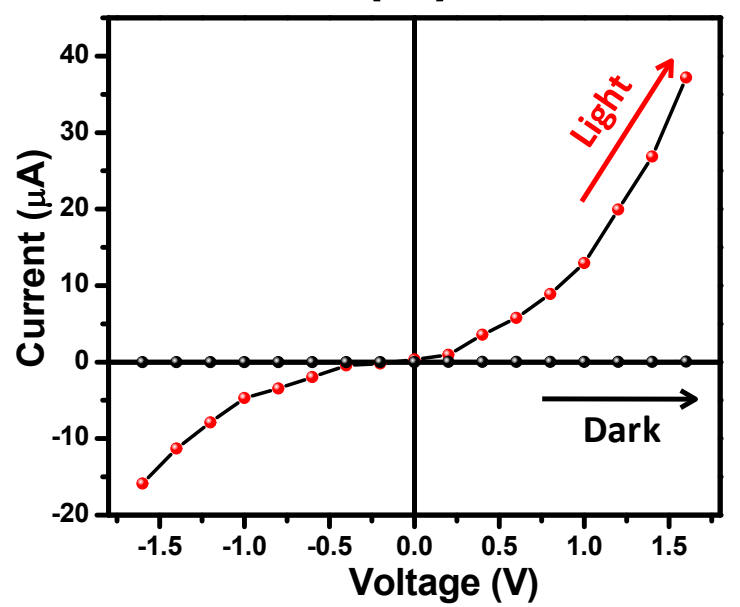

Figure S8. $\mathrm{I}-\mathrm{V}$ curves of composites (A) CdSe-CsPbBr ${ }_{1.5} \mathrm{I}_{1.5}$ and (B) $\mathrm{CdSe}-\mathrm{CsPbBr}_{2}$. 


\section{References:}

(1) Ravi, V. K.; Markad, G. B.; Nag, A., Band Edge Energies and Excitonic Transition Probabilities of Colloidal $\mathrm{CsPbX}_{3}(\mathrm{X}=\mathrm{Cl}, \mathrm{Br}, \mathrm{I})$ Perovskite Nanocrystals. ACS Energy Lett. 2016, $1,665-671$.

(2) Protesescu, L.; Yakunin, S.; Bodnarchuk, M. I.; Krieg, F.; Caputo, R.; Hendon, C. H.; Yang, R. X.; Walsh, A.; Kovalenko, M. V., Nanocrystals of Cesium Lead Halide Perovskites $\left(\mathrm{CsPbX}_{3}, \mathrm{X}=\mathrm{Cl}, \mathrm{Br}\right.$, and I): Novel Optoelectronic Materials Showing Bright Emission with Wide Color Gamut. Nano Lett. 2015, 15, 3692-3696. 